\title{
Bloqueo caudal más anestesia general balanceada en un paciente con pentalogía de Cantrell, presentación de un caso clínico
}

\section{Caudal block plus inhaled general anesthesia in a patient with pentalogy of Cantrell, presentation of a clinical case}

Silvia Zepeda Olivera ${ }^{1,2, *}$, Mariana Rosas de la Llave², David Ricardo González Perilla², Jorge Ariel Nieto Ordoñez², Ana Karen Méndez García1,a

Hospital el Niño Poblano. Puebla, México.

2 Benemérita Universidad Autónoma de Puebla. Puebla, México.

Conflicto de interés: no se reporta ninguno.

Fecha de recepción: 21 de febrero de 2021 / Fecha de aceptación: 12 de mayo de 2021

\begin{abstract}
The pentalogy of Cantrell is a disorder characterized by congenital abnormalities in the abdominal wall, lower sternum, anterior diaphragm, diaphragmatic pericardium, and cardiac anomalies. It is a rare disease with 250 cases registered around the world. The anesthetic implications will require a specialized management given the ventilatory mechanics and cardiac function which are compromised by the disease in the newborn. We present the case of a female patient with pentalogy of Cantrell without prenatal diagnosis, who had an operative procedure to correct patent ductus arteriosus and abdominal mesh placement under balanced general anesthesia with sevoflurane and fentanyl plus caudal block. This case is reported to provide our experience in the anesthetic management of this type of patients.
\end{abstract}

Key words: Pentalogy of Cantrell, congenital disease, omphalocele.

\section{RESUMEN}

La pentalogía de Cantrell es una enfermedad caracterizada por anormalidades congénitas de la pared abdominal supraumbilical, esternón inferior, diafragma, pericardio diafragmático y anomalías cardiacas. Se trata de una enfermedad rara con 250 casos registrados alrededor del mundo. Las implicaciones anestésicas requieren de un manejo especializado debido a la mecánica ventilatoria y función cardíaca que se encuentran comprometidas en el recién nacido. Se presenta el caso de una recién nacida portadora de pentalogía de Cantrell, no diagnosticada prenatalmente, quien fue sometida a corrección de ductus arterioso persistente y colocación de malla abdominal bajo anestesia general balanceada con sevofluorano y fentanilo más bloqueo caudal. Se reporta el presente caso para brindar nuestra experiencia en el manejo anestésico de este tipo de pacientes.

Palabras clave: Pentalogía de Cantrell, enfermedad congénita, onfalocele. 


\section{Introducción}

ames R. Cantrell definió esta pentalogía en 1958, las anormalidades incluyen: un defecto de la pared abdominal supraumbilical, la parte inferior del esternón, agenesia de la porción anterior del diafragma, ausencia de la cara diafragmática del pericardio y malformaciones cardiacas[1],[2]. La incidencia se estima de 1 en 200.000 nacimientos[3]. Se trata de una enfermedad rara con apenas 250 casos registrados alrededor del mundo hasta 2009[1],[4], de los cuales 72\% se han reportado en los Estados Unidos y Europa, el resto en Asia[4] no se cuenta con estadísticas para Latinoamérica. Patología cuya mortalidad asciende al $63 \%$ y una supervivencia máxima posterior a la cirugía de 9 meses[5].

El tratamiento es estrictamente quirúrgico y su corrección es intrincada debido al amplio espectro y complejidad de las anormalidades[1].

Las implicaciones anestésicas representan todo un reto, ya que el abordaje ventilatorio y hemodinámico demandan de una habilidad avanzada en el manejo del paciente neonato debido a la inmadurez pulmonar, los defectos cardiacos y la alta presión intraabdominal posterior al cierre de las paredes torácica y abdominal[5].

En todos los pacientes se realizará una intubación difícil debido a la posición anterior y cefálica del corazón, la hipoplasia pulmonar y el distrés respiratorio debido a la hipoplasia torácica.

Las prioridades críticas en el perioperatorio serán la pérdida de líquidos, la elevación de las presiones intratorácica e intraabdominal al internalizar las estructuras ectópicas, la reparación de la pared abdominal y el colapso cardiaco por la presión en los grandes vasos y cámaras cardiacas.

\section{Caso clínico}

Recién nacida femenina de un día de vida extrauterina, producto a término, sin antecedentes heredofamiliares de importancia. Control prenatal irregular, ultrasonido fetal al $7^{\circ}$ mes reportado sin alteraciones; obtenida mediante parto eutócico en medio hospitalario, APGAR 8-9, Silverman 0, peso $2.620 \mathrm{~g}$,

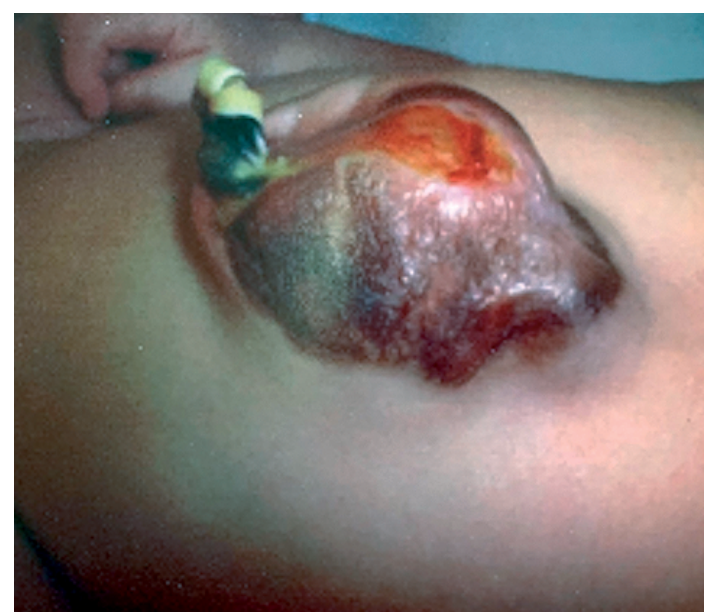

Figura 1. Paciente con onfalocele toracoabdominal. talla $42 \mathrm{~cm}$. A la exploración física neonatal se aprecia onfalocele por lo que es trasladada a tercer nivel de atención hospitalaria para valoración y corrección quirúrgica.

Al ingreso presenta onfalocele toracoabdominal (Figura 1), pulmones con murmullo vesicular disminuido, ruidos cardiacos con presencia de soplo holosistólico en todo el precordio; abdomen con defecto a nivel de la línea media de aproximadamente $5 \times 4 \mathrm{~cm}$, a través del cual se observa masa pulsátil cubierta por peritoneo, resto de exploración sin alteraciones aparentes.

Se realiza radiografía toracoabdominal y ultrasonido abdominal en los que se confirma protrusión de ápex cardiaco (Figuras 2 y 3). Laboratorios dentro de parámetros normales.

Paciente con probable pentalogía de Cantrell, por lo que se ingresa de urgencia a sala de quirófano para colocación de malla y corrección de ductus arterioso persistente.

Plan anestésico: ASA IV. Bloqueo caudal más anestesia general balanceada con sevoflurano y fentanilo.

Ingresa a quirófano reactiva, con catéter venoso periférico permeable, monitorización no invasiva (Tabla 1), sala a $27^{\circ} \mathrm{C}$ y colchón térmico (Figura 4). Medicación preanestésica: atropina 25 mcg. Inducción: fentanil $5 \mathrm{mcg}$, propofol $5 \mathrm{mg}$, vecuronio 300 mcg, asistencia con bolsa-mascarilla por 3 minutos, intubación orotraqueal con cánula 3,5, conectándose a sistema circular pediátrico, sin embargo, no tolera asistencia ventilatoria mecánica y se asiste manualmente.

Bloqueo caudal con aguja Tuohy $17 \mathrm{G}$, anestésico local bupivacaína 0,2\%, teniendo el dermatoma T4 como meta por el tipo de intervención y al tratarse de un abordaje caudal, se decide calcular la dosis en $\mathrm{ml} / \mathrm{kg}[8]$, administrando un volumen total de $4 \mathrm{ml}$, dicha titulación también permitió emplear dosis bajas de halogenados y opiáceos. Se coloca catéter epidural el cual no se emplea en intraoperatorio sino para manejo analgésico postoperatorio.

Mantenimiento: sevofluorano 1 vol\%, oxígeno/aire a $3 \mathrm{l}$ min, $\mathrm{FiO}_{2} 75 \%$. Fentanil $10 \mathrm{mcg}$ al presentar taquicardia y elevación de la tensión arterial por estímulo quirúrgico (dosis total $15 \mathrm{mcg}$ ).

Al realizar cierre de conducto arterioso, presenta desaturación de $\mathrm{O}_{2}$ y bradicardia por lo que se inicia dopamina a 5 $\mathrm{mcg} / \mathrm{kg} / \mathrm{min}$, logrando estabilización hemodinámica; posteriormente, al internalizar onfalocele, la tensión arterial desciende a

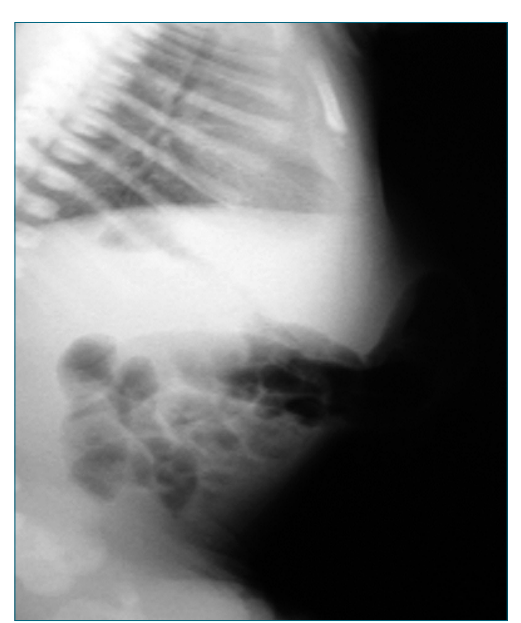

Figura 2. Radiografía toracoabdominal donde se observa malformación esternal. 


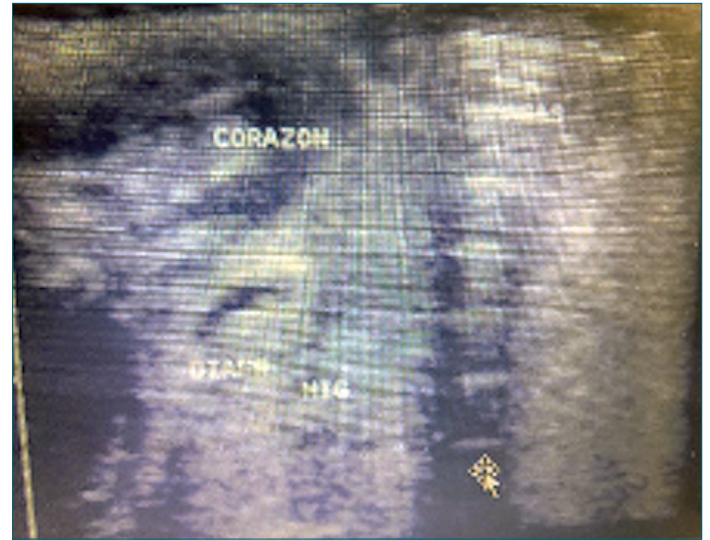

Figura 3. Ultrasonido abdominal.

$40 / 20 \mathrm{mmHg}$ y la saturación de $\mathrm{O}$, disminuye a $72 \%$, se incrementa infusión de dobutamina a $10 \mathrm{mcg} / \mathrm{kg} / \mathrm{min}$, estabilizando paciente; se decide colocar malla protectora abdominal y dar por terminado el procedimiento quirúrgico debido a la labilidad hemodinámica intraoperatoria a pesar del apoyo farmacológico.

Se traslada paciente a la unidad de cuidados intensivos neonatales, intubada, con signos vitales en parámetros adecuados, con infusión de dobutamina $10 \mathrm{mcg} / \mathrm{kg} / \mathrm{min}$, se indica analgesia peridural con bupivacaina $100 \mathrm{mcg} / \mathrm{kg} /$ día más fentanil endovenoso $2 \mathrm{mcg} / \mathrm{kg} / \mathrm{día}$, a las 24 h se retira catéter epidural sin incidentes ni complicaciones. Se continúa analgesia endovenosa con fentanil dosis $2 \mathrm{mcg} / \mathrm{kg} / \mathrm{día}$; al $5^{\circ}$ día se inicia norepinefrina a $100 \mathrm{mcg} / \mathrm{kg} / \mathrm{min}$ y administración de oxido nítrico por presentar datos de hipertensión pulmonar; al $6^{\circ}$ día se agregan antibióticos de amplio espectro para manejo inicial de sepsis neonatal, falleciendo al $8^{\circ}$ día; la necropsia corrobora el diagnóstico de Pentalogía de Cantrell.

\section{Discusión}

El reporte de este caso obedece a la baja incidencia de esta entidad a nivel mundial que no permite contar con bibliografía extensa enfocada en el manejo anestésico.

Se eligió técnica combinada la cual nos brindó la ventaja de disminuir los requerimientos de anestésicos inhalados y opiáceos de mantenimiento. En la literatura no encontramos ningún reporte que coincida con la técnica empleada, siendo éste nuestro aporte en el manejo anestésico en este tipo de pacientes.

El catéter peridural caudal ofreció la posibilidad de garantizar analgesia postoperatoria durante las primeras $24 \mathrm{~h}$ al combinarse con fentanilo intravenoso.

La paciente presentaba ectopia cordis, pronosticándole una mortalidad del 50\% como lo refiere la literatura. Adicionalmente, se agregó sepsis neonatal e hipertensión pulmonar, ensombreciendo su pronóstico.

Son necesarias más publicaciones referentes al tema que permitan establecer un consenso acerca del abordaje anestésico óptimo.

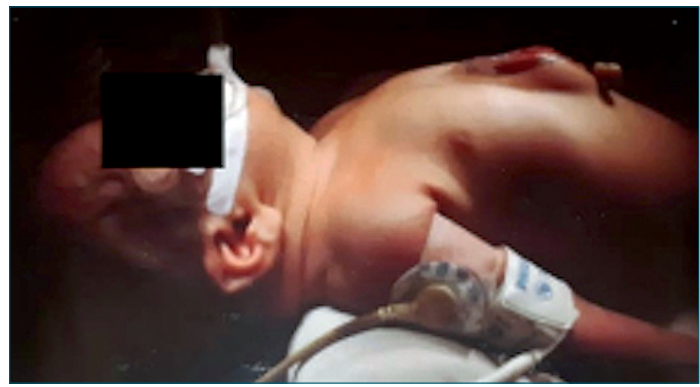

Figura 4. Paciente intubada con pentalogía de Cantrell.

\begin{tabular}{lcc} 
& Tabla 1. Signos vitales \\
\hline Ingreso & Quirófano & UCIN \\
$\begin{array}{l}\text { Tensión arterial } \\
(\mathrm{mmHg})\end{array}$ & $80 / 45$ & $95 / 42$ \\
$\begin{array}{l}\text { Frecuencia cardiaca } \\
(\text { Ipm) }\end{array}$ & 143 & 116 \\
$\begin{array}{l}\text { Frecuencia respiratoria } \\
(\text { rpm })\end{array}$ & 45 & 43 \\
$\begin{array}{l}\text { Temperatura } \\
\left({ }^{\circ} \mathrm{C}\right)\end{array}$ & 36,7 & 36,3 \\
\hline
\end{tabular}

\section{Referencias}

1. Williams AP, Marayati R, Beierle EA. Pentalogy of Cantrell. Semin Pediatr Surg. 2019 Apr;28(2):106-10. https://doi.org/10.1053/j. sempedsurg.2019.04.006 PMID:31072457

2. Suehiro K, Okutani R, Ogawa S, Nakada K, Shimaoka H, Ueda $M$, et al. Perioperative management of a neonate with Cantrell syndrome. J Anesth. 2009;23(4):572-5. https://doi.org/10.1007/ s00540-009-0785-9 PMID:19921368

3. Kylat RI. Complete and Incomplete Pentalogy of Cantrell. Children (Basel). 2019 Oct;6(10):109. https://doi.org/10.3390/children6100109 PMID:31590448

4. Grigore M, Micu R, Matasariu R, Duma O, Chicea AL, Chicea R. Cantrell syndrome in the first trimester of pregnancy: imagistic findings and literature review. Med Ultrason. 2020 May;22(2):189-96. https://doi.org/10.11152/mu-2316 PMID:32190852

5. Costa JV, Melonio CE, Vieira CB, Oliveira CM, Leal PC, Servín ET, et al. da Silva Costa JV y cols. Anesthesia for surgical repair of the pentalogy of Cantrell: case report. Braz J Anesthesiol. 2019 Mar;69(3):322-5. https://doi.org/10.1016/j.bjane.2018.09.011.

6. Cuesta-Guardiola T, Aluja-Méndez A, Pérez Fernández-Pacheco R, Gámez-Alderete F, Ortiz-Quintana L, De León-Luis J. Diagnóstico prenatal de pentalogía de Cantrell. Progresos de Obstetricia y Ginecología; 2015. https://doi.org/10.1016/j.pog.2015.07.010.

7. Nichols JH, Nasr VG. Sternal malformations and anesthetic management. Paediatr Anaesth. 2017 Nov;27(11):1084-90. https:// doi.org/10.1111/pan.13253 PMID:29030926

8. Forestier J, Castillo P, Finnbogason T, Lundblad M, Eksborg S, Lönnqvist PA. Volumes of the spinal canal and caudal space in children zero to three years of age assessed by magnetic resonance imaging: implications for volume dosage of caudal blockade. Br J Anaesth. 2017 Nov;119(5):972-8. https://doi.org/10.1093/ bja/aex280 PMID:29028948 